\title{
Short lever arm, bipedicular handlebar construct for correction of acute angular kyphosis in spondylodiscitis-induced kyphotic deformity: illustrative case
}

\author{
${ }^{*}$ Meng Huang, MD, ${ }^{1,2}$ lahn Cajigas, MD, PhD, ${ }^{1}$ and Steven Vanni, DO ${ }^{1}$ \\ ${ }^{1}$ Department of Neurosurgery, University of Miami, Miami, Florida; and ${ }^{2}$ Department of Neurosurgery, Houston Methodist Neurological Institute, Houston, Texas
}

BACKGROUND Pyogenic spondylodiscitis diminishes spinal structural integrity via disruption of the anterior and middle column, sometimes further compounded by iatrogenic violation of the posterior tension band during initial posterior decompressive surgeries. Although medical management is typically sufficient, refractory infection or progressive deformity may require aggressive debridement and reconstructive arthrodesis. Although anterior debridement plus reconstruction with posterior stabilization is an effective treatment option, existing techniques have limited efficacy for correcting focal deformity, leaving patients at risk for long-term sagittal imbalance, pain, and disability.

OBSERVATIONS The authors present a case of chronic lumbar pyogenic spondylodiscitis in a patient in whom initial surgical debridement failed and pronounced angular kyphosis and intractable low back pain developed. A novel bipedicular handlebar construct was used to achieve angular correction of the kyphosis through simultaneous anterior interbody grafting and posterior instrumentation with the patient in the lateral position.

LESSONS Leveraging both pedicle screws at the same level to transmit controlled corrective distraction forces through the segment allows for kyphosis correction without relying on long posterior constructs for cantilever reduction. Simultaneous anterior reconstruction with a posterior short lever arm, bipedicular handlebar construct is an effective technique for achieving high angular correction during circumferential reconstructive approaches to postinfectious focal kyphotic deformities.

https://thejns.org/doi/abs/10.3171/CASE21190

KEYWORDS kyphosis; deformity; instrumentation; manipulation; spondylodiscitis

Surgical intervention for pyogenic spondylodiscitis is a wellestablished treatment modality for cases refractory to conservative management. ${ }^{1,2}$ Instrumented spinal fusion is typically required when destruction of the anterior and middle columns result in de novo instability or deformity or when postlaminectomy kyphosis develops after initial decompressive surgery. ${ }^{3-5}$ Anterior column debridement with autologous structural graft reconstruction has been the preferred standard of treatment. ${ }^{3,6,7}$ Several recent studies have proposed the minimally invasive lateral transpsoas approach for reconstructive arthrodesis and infectious source control. ${ }^{8-10}$ In these three studies, patients underwent anterior lateral transpsoas debridement with interbody grafting/reconstruction as a stand-alone construct or supplemented with posterior pedicle screw fixation. Only in the case of supplemental posterior pedicle screw fixation was there a modest degree of segmental kyphosis improvement. ${ }^{8}$ Here, we present a unique technique for correction of a severe kyphotic deformity implemented in the lateral position with simultaneous lateral transpsoas reconstruction and cantilever distraction through a short segment posterior construct. This technique facilitated high angular correction and minimized overall construct length.

\section{Illustrative Case}

A 65-year-old man with a past medical history of hyperlipidemia had sustained a recent fall that caused an elbow laceration and deep tissue infection requiring surgical debridement and treatment with intravenous (IV) antibiotics. The patient presented several weeks later to an outside institution because of the development of severe low back pain (worse with movement) and lumbar

ABBREVIATIONS $C T=$ computed tomography; IV = intravenous; MRI = magnetic resonance imaging; $X L I F=$ extreme lateral interbody fusion. INCLUDE WHEN CITING Published June 14, 2021; DOI: 10.3171/CASE21190.

SUBMITTED March 29, 2021. ACCEPTED April 5, 2021

${ }^{*}$ M.H. and I.C. contributed equally to this work.

(c) 2021 The authors, CC BY-NC-ND 4.0 (http://creativecommons.org/licenses/by-nc-nd/4.0/). 

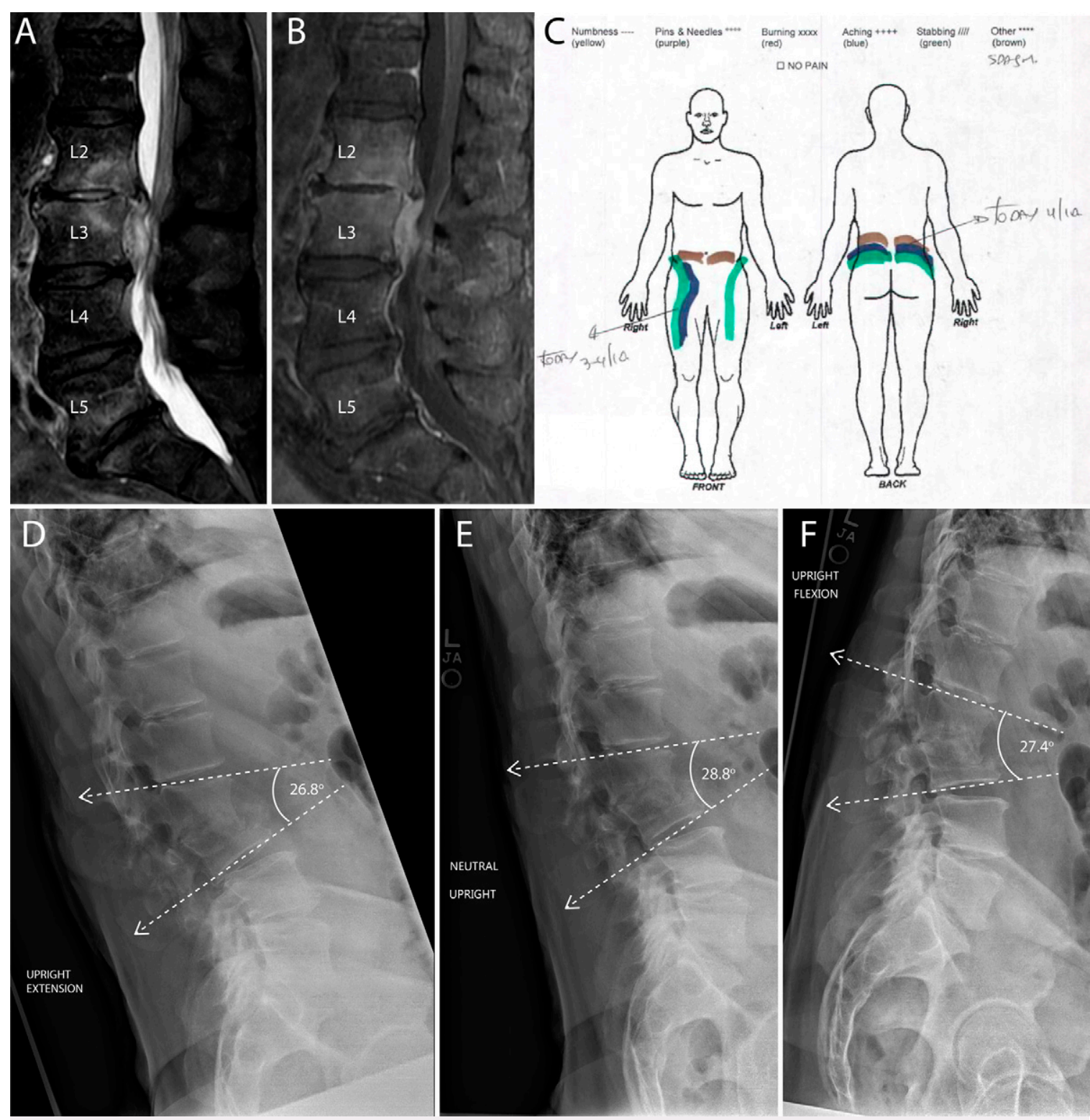

FIG. 1. A: Sagittal MRI short tau inversion recovery (STIR) sequence showing significant inflammation of the L2-3 disc along with the adjacent vertebral body and endplates. B: Sagittal postgadolinium sequence demonstrates additional presence of a ventral epidural abscess. C: Pain diagram 6 weeks after initial decompressive surgery. Preoperative standing radiographs with dynamic extension (D), neutral (E), and flexion (F) views and segmental angles.

radiculopathy. Lumbar magnetic resonance imaging (MRI) showed L2-3 discitis with an associated epidural abscess without any gross deformity (Fig. 1A and B). The patient initially underwent bilateral L2-3 laminotomy for debridement, and intraoperative cultures grew Streptococcus mitis. He had no prior history or current evidence of odontogenic disease and subsequently received 6 weeks of IV antibiotic therapy. Despite initial symptomatic improvement and downtrending inflammatory markers, the patient developed worsening low back pain and groin pain (right side worse than left; Fig. 1C). Interval lateral radiographs showed significant endplate erosion at L2 with the development of vertebral body subsidence and an acute kyphotic deformity. On upright lateral radiographs, the Cobb angle at L2-3 was $26^{\circ}$ and did not vary between flexion and extension (Fig. 1D-F). On supine computed tomography (CT) imaging, the kyphotic deformity corrected by only approximately $5^{\circ}$, indicating a relatively rigid deformity (Fig. 2). To correct the acute kyphotic deformity without using a long posterior construct for cantilever reduction, we developed a transverse short lever arm, bipedicular handlebar construct for transmission of corrective forces using only pedicle screws placed at $\mathrm{L} 2$ and $\mathrm{L} 3$, which we detail below.

\section{Technique}

After a standard preoperative protocol and induction of general anesthesia, the patient was placed prone on a Jackson table. After 

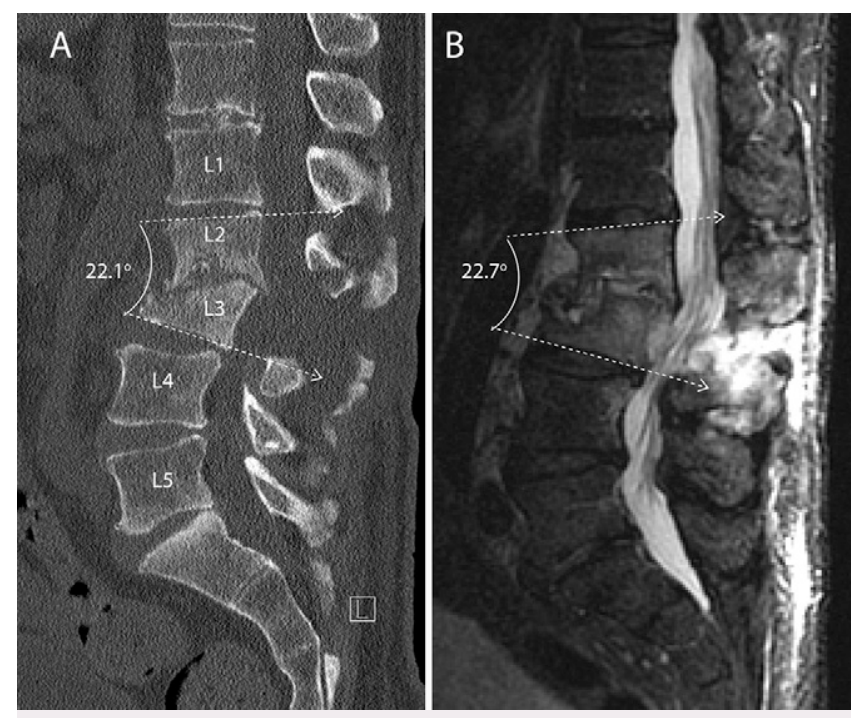

FIG. 2. Preoperative supine imaging with $C T(\mathbf{A})$ and $M R I S T I R$ sequence (B) with segmental angle.

sterile prepping and draping, the prior posterior incision was opened and the L2-3 and L1-2 facet complexes were exposed. Pedicle screws were placed bilaterally at L2 and L3 using a fluoroscopeassisted free-hand technique. The screws were directed away from the osteolytic defects in the superior aspect of the $L 3$ vertebral body and inferior aspect of the L2 vertebral body. The facet joint of L2-3 was undisturbed; however, in cases in which a larger degree of correction is required, osteotomies to remove the facet joints could additionally be performed at this stage. Two rods were then contoured and secured across the pedicle screws transversely so that both L2 and L3 screws were interconnected (Fig. 3A and B). The wound was stapled closed, and the patient was repositioned with the lateral left side up in the standard fashion for a lateral transpsoas approach. Fluoroscopy was used to target the level of interest; after sterile prepping and draping, a small 4-cm incision was made over the L2-3 disc space. The target disc was accessed using the standard extreme lateral interbody fusion (XLIF; NuVasive, Inc.) technique, and the retractor was secured using two bedmounted rigid arms.

Both chronic phlegmon and active frank purulent material were encountered within the disc space and the adjacent vertebral bodies. Curettes and rongeurs were used to remove all pathological tissue. Once satisfactory discectomy and partial corpectomy had been completed, the posterior incision was reopened, and the handlebar rods were exposed and engaged with heavy-duty rod holders. Cadaveric ilium was used as structural allograft to fill the anterior vertebral defect. While placing the structural allograft via the lateral corridor, posterior compression forces were applied to the bipedicular handlebar construct to facilitate anterior distraction and kyphosis
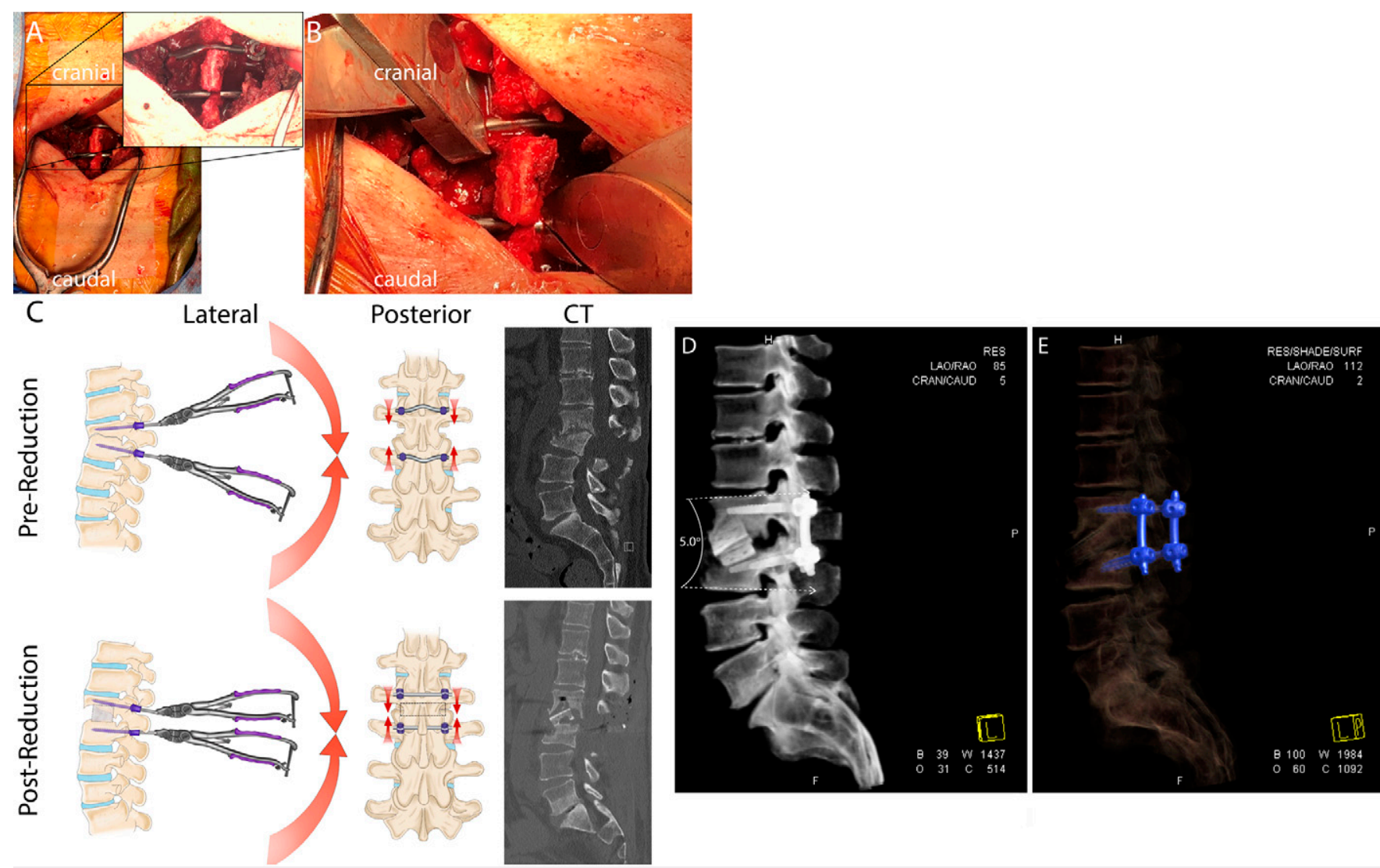

FIG. 3. A and B: Intraoperative photographs of construct. C: Illustrations demonstrating rod holder attachment to construct with patient in lateral position and force vector transmission, along with midsagittal CT scans before and after correction. D and E: Postoperative routine CT scans showing two- and three-dimensional reconstructions with final segmental angle. 
correction (Fig. 3C). After satisfactory near-complete correction of kyphosis and graft placement, the posterior transverse rods were removed and replaced with standard craniocaudal rods between $\mathrm{L} 2$ and $\mathrm{L} 3$ bilaterally to fixate and secure the segment. Both the lateral and posterior incisions were closed in a standard layered fashion.

The patient experienced immediate improvement in his preoperative pain and began mobilizing with physical therapy on postoperative day 1. A routine postoperative CT demonstrated significant improvement in segmental kyphosis and hardware in good position (Fig. 3D and E). By postoperative day 4 , the patient had been cleared for discharge home but remained in the hospital pending final intraoperative tissue cultures for determination of the IV antibiotic regimen. However, intraoperative cultures ultimately yielded no growth. The patient was discharged home on postoperative day 8 with a peripherally inserted central catheter and IV ceftriaxone, with a 6-week treatment plan based on his history of $S$ mitis and antibiotic sensitivities of the outside-hospital specimen. The patient has recovered well postoperatively, with maintained clinical improvement and radiographic evidence of solid arthrodesis through the anterior column at the 13-month follow-up.

\section{Discussion}

Anterior debridement and reconstruction with or without posterior supplemental fixation is the accepted standard for surgical treatment of spondylodiscitis with deformity. ${ }^{2,3,11-14}$ With the popularization of the minimally invasive lateral transpsoas approach for trauma and degenerative conditions of the spine, several authors have reported their success in treating spondylodiscitis via this approach. $^{8-10}$ However, despite their reports of clinical outcomes and complications in the short-term follow-up, available studies have not placed major emphasis on the degree of deformity correction ultimately achieved. In their cohort of 6 patients with lumbar spondylodiscitis treated with stand-alone XLIF with anterior plating, Patel et al. did not report radiographic outcomes with respect to the pre- and postoperative segmental Cobb angle. ${ }^{9}$ Timothy et al. reported that the they were unable to address correction of any preoperative kyphotic deformity in 14 patients with thoracic and lumbar spondylodiscitis treated with stand-alone XLIF. ${ }^{10}$ However, no significant progressive deformity occurred postoperatively. Lastly, Blizzard et al. reported a mean improvement of $9.2^{\circ}$ of segmental kyphosis
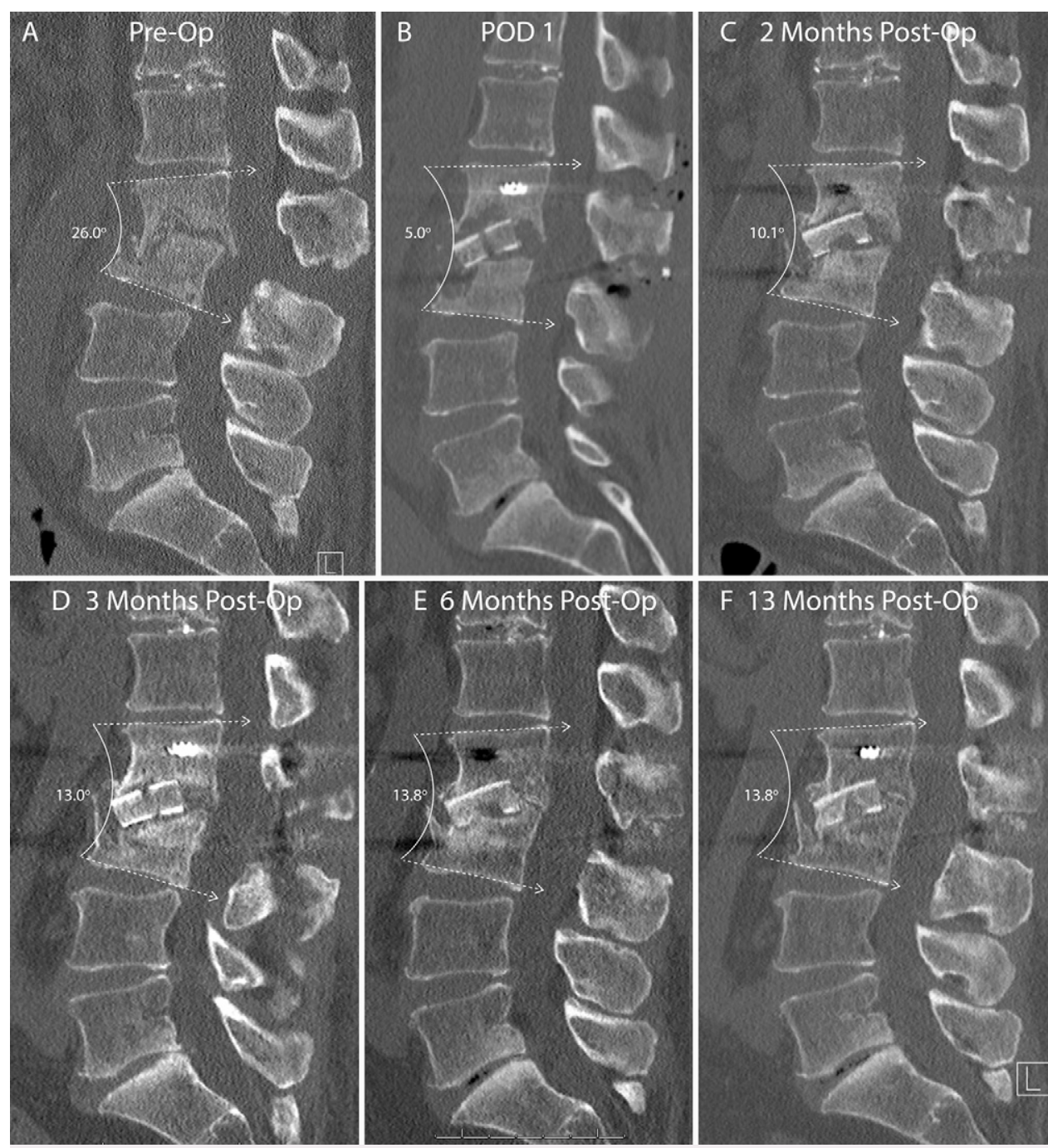

FIG. 4. A-F: Changes in segmental correction across time. POD = postoperative day; Post-Op = postoperative; Pre-Op = preoperative. 


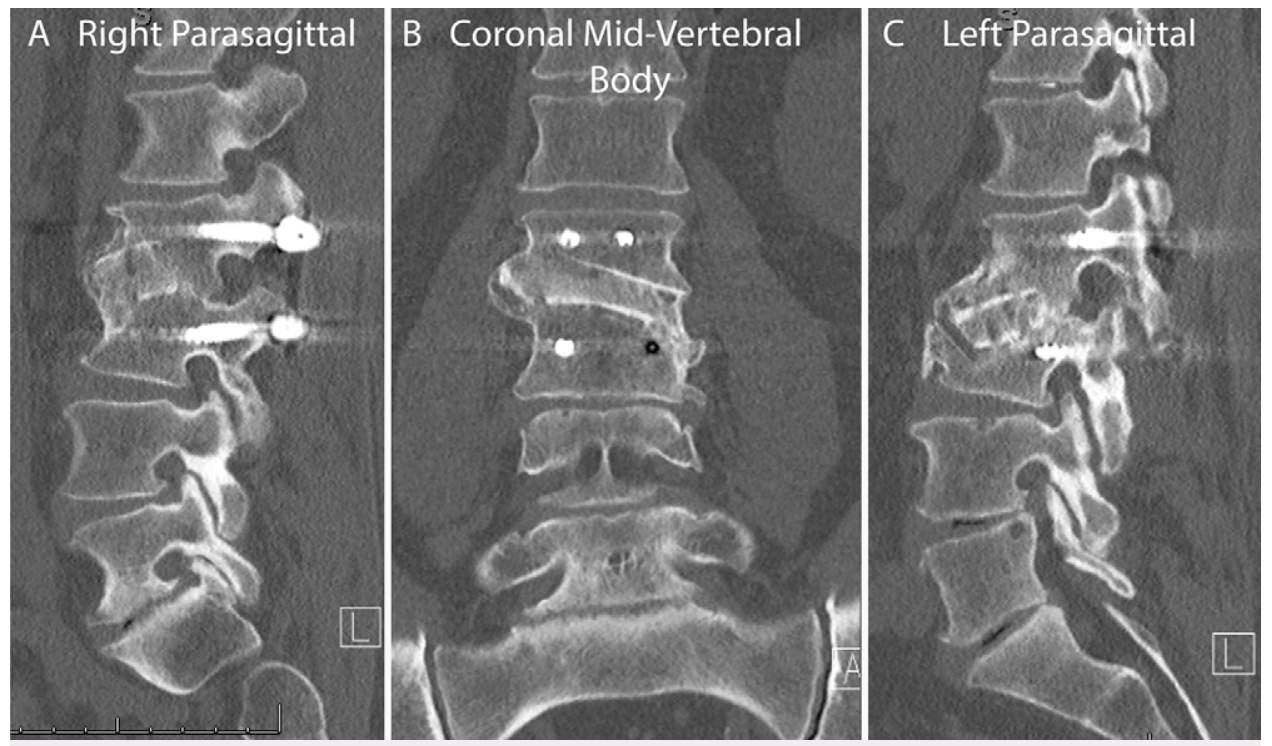

FIG. 5. CT of right parasagittal (A), coronal midvertebral body (B), and left parasagittal (C) regions showed circumferential arthrodesis at 13-month follow-up.

in their series of 11 patients. Notably, all patients underwent prone positioning and received a supplemental posterior pedicle screw after the anterior reconstruction. ${ }^{8}$

\section{Observations}

In our case, the patient had severe focal angular kyphosis of $26^{\circ}$ on upright weight-bearing radiographs, with minimal movement between flexion and extension views. On the supine image, the kyphosis corrected by only $5^{\circ}$, indicating a relatively rigid deformity. Given the prior evidence by Timothy et al. ${ }^{10}$ of the inability to improve kyphotic deformity with the anterior approach alone and the study by Blizzard et al. ${ }^{8}$ demonstrating segmental kyphosis improvement with the addition of posterior pedicle screw supplementation, we believed that a circumferential approach was necessary for effective deformity correction rather than a stand-alone anterior construct. We also believed that an anterior approach followed by a posterior approach would have been suboptimal in correcting this relatively high angle deformity. Given the total endplate destruction and pronounced erosion of the subchondral cancellous bone of the adjacent vertebral bodies, distraction forces applied across the defect, either by expansion of an interbody device or directly by distraction/spreader devices, would have likely resulted in telescoping into the vertebral bodies with the failure to achieve sagittal plane correction. We also thought that relying predominately on posterior instrumentation for correction would have required a long construct of at least two levels above and below to impart and sustain the cantilever forces necessary for reduction of this degree of acute angular kyphosis.

Therefore, to effectively correct the deformity and simultaneously minimize the construct length, we opted to implement this novel simultaneous anterior-posterior technique. By capturing both pedicle screws of the same level with a transverse rod, we were able to safely transmit distractive forces across the segment in the sagittal plane. With concomitant impaction of a custom-cut oversized tricortical ilium allograft, this technique allowed us to achieve our surgical goal with only a single-level construct. Our final segmental angle was $5^{\circ}$ of kyphosis, improved from $26^{\circ}$, for an overall segmental correction of $21^{\circ}$. The construct settled and lost $5.1^{\circ}$ of correction by 2 months, for a total segmental kyphosis of $10.1^{\circ}$ and a residual correction of $15.9^{\circ}$. This further settled to $13^{\circ}$ of kyphosis at 3 months and finally to $13.8^{\circ}$ at 6 months, with $12.2^{\circ}$ of ultimate segmental correction. There was no further change in segmental angle at the 13-month follow-up compared to that at 6 months (Fig. 4). The 13-month postoperative CT confirmed circumferential evidence of arthrodesis (Fig. 5).

\section{Lessons}

The short lever arm, bipedicular handlebar construct is an effective way to optimize control of one spinal segment and transmit distractive forces. When used with the patient in a lateral position for simultaneous anterior reconstruction and posterior fixation, large angular corrections can be achieved without a long posterior construct. Although additional cases are needed to assess the reproducibility of this technique, this initial case demonstrates compelling evidence to support further clinical implementation of this technique, which can be expanded to other spinal indications, including trauma and metastatic neoplasm.

\section{Acknowledgments}

We acknowledge Roberto Suazo for his assistance with medical illustrations.

\section{References}

1. Bydon M, De la Garza-Ramos R, Macki M, et al. Spinal instrumentation in patients with primary spinal infections does not lead to greater recurrent infection rates: an analysis of 118 cases. World Neurosurg. 2014;82(6):e807-e814.

2. Chen WH, Jiang LS, Dai LY. Surgical treatment of pyogenic vertebral osteomyelitis with spinal instrumentation. Eur Spine J. 2007; 16(9):1307-1316. 
3. Hsieh PC, Wienecke RJ, O'Shaughnessy BA, et al. Surgical strategies for vertebral osteomyelitis and epidural abscess. Neurosurg Focus. 2004;17(6):E4.

4. Noh SH, Zhang HY, Lim HS, et al. Decompression alone versus fusion for pyogenic spondylodiscitis. Spine J. 2017;17(8):1120-1126.

5. Than KD, Mummaneni PV. Spinal instrumentation in the setting of primary infection. World Neurosurg. 2014;82(6):e713-e714.

6. Acosta FL Jr, Chin CT, Quiñones-Hinojosa A, et al. Diagnosis and management of adult pyogenic osteomyelitis of the cervical spine. Neurosurg Focus. 2004;17(6):E2.

7. Zeng $Y$, Cheng $P$, Tan J, et al. Comparison of three surgical approaches for thoracolumbar junction (T12-L1) tuberculosis: a multicentre, retrospective study. BMC Musculoskelet Disord. 2019;20(1):524.

8. Blizzard DJ, Hills CP, Isaacs RE, Brown CR. Extreme lateral interbody fusion with posterior instrumentation for spondylodiscitis. J Clin Neurosci. 2015;22(11):1758-1761.

9. Patel NB, Dodd ZH, Voorhies J, Horn EM. Minimally invasive lateral transpsoas approach for spinal discitis and osteomyelitis. J Clin Neurosci. 2015;22(11):1753-1757.

10. Timothy J, Pal D, Akhunbay-Fudge $C$, et al. Extreme lateral interbody fusion (XLIF) as a treatment for acute spondylodiscitis: Leeds spinal unit experience. J Clin Neurosci. 2019;59:213-217.

11. Lin Y, Li F, Chen W, et al. Single-level lumbar pyogenic spondylodiscitis treated with mini-open anterior debridement and fusion in combination with posterior percutaneous fixation via a modified anterior lumbar interbody fusion approach. J Neurosurg Spine. 2015;23(6):747-753.

12. Mann S, Schütze M, Sola S, Piek J. Nonspecific pyogenic spondylodiscitis: clinical manifestations, surgical treatment, and outcome in 24 patients. Neurosurg Focus. 2004;17(6):E3.
13. Nakase H, Matsuda R, Tamaki R, et al. Two-stage management for vertebral osteomyelitis and epidural abscess: technical note. Neurosurgery. 2006;58(6):E1219.

14. Sundararaj GD, Amritanand R, Venkatesh K, Arockiaraj J. The use of titanium mesh cages in the reconstruction of anterior column defects in active spinal infections: can we rest the crest? Asian Spine J. 2011;5(3):155-161.

\section{Disclosures}

Dr. Vanni receives royalty payments from NuVasive, Globus, and Spine Wave and serves in a consultant position with RTI Surgical. Dr. Huang serves in consultant positions for Integrity Implants, Depuy Synthes, and Joimax, but did not at the time this study was written.

\section{Author Contributions}

Conception and design: Huang, Vanni. Acquisition of data: all authors. Analysis and interpretation of data: Huang, Vanni. Drafting the article: Huang, Cajigas. Critically revising the article: all authors. Reviewed submitted version of manuscript: all authors. Approved the final version of the manuscript on behalf of all authors: Huang. Administrative/ technical/material support: Cajigas. Study supervision: Vanni.

\section{Correspondence}

Meng Huang: Houston Methodist Neurological Institute, Houston, TX mhuang@houstonmethodist.org. 